\title{
Idiopathic pseudotumours of the pterygopalatine fossa: case series and a narrative review*
}

\author{
Muhammad Kamaal Khan', Jason Powell', Mohamed Reda ElBadawey ${ }^{1,2}$, \\ Sean Carrie
}

' Department of Otolaryngology - Head and Neck Surgery, Freeman Hospital, Newcastle upon Tyne, United Kingdom

${ }^{2}$ Department of Otolaryngology, Tanta University, Egypt
Rhinology Online, Vol 2: 14 - 20, 2019

http://doi.org/10.4193/RHINOL/18.028

*Received for publication:

June 28, 2018

Accepted: January 17, 2019

Published: January 25, 2019

\begin{abstract}
Background: Idiopathic pseudotumour (IP) is a non-specific, non-neoplastic, inflammatory process without identifiable local or systemic causes. IP in the head and neck region present diagnostic and management issues.
\end{abstract}

Methodology: Retrospective case series and literature review.

Results: We describe clinical history, examination, laboratory investigations and image findings of three patients, all males who presented with facial pain and ophthalmoplegia. Scans revealed lesions in the pterygopalatine fossa. The diagnosis of idiopathic pseudotumour was confirmed by histopathology. We discuss their treatment pathways and prognosis.

Conclusions: Idiopathic pseudotumours in the head and neck region especially those involving anterior skull base are rare. Understanding their presentation and characteristics might help an unsuspecting otorhinolaryngologist in the diagnosis and treatment of these lesions.

Key words: Skull base, inflammatory pseudotumor, tumour, diagnosis, treatment

\section{Introduction}

Idiopathic pseudotumour (IP) is a rare nonspecific, non-neoplastic inflammatory process without identifiable local or systemic cause ${ }^{(1)}$. IP is not a single entity but an umbrella term for any nonspecific chronic inflammatory expansile lesion ${ }^{(2)}$. Whilst more frequently in the lungs and orbit, they have also been described in other anatomic sites including the peritoneum, extremities, temporal bones, salivary glands, upper aerodigestive tracts and the skull base ${ }^{(3)}$. The head and neck lesions are rare, accounting for $15 \%$ of all sites with $<5 \%$ involving extra-orbital sites ${ }^{(2,4)}$. Busse and Hocheim first described a form of head and neck IP in 1903. The term 'pseudotumour' was first coined by Birch-Hirschfield in 1905 because of its locally aggressive but non-neoplastic nature ${ }^{(1)}$.

IP can affect any age group and have been described in both sexes. Unilaterality is the norm but synchronous lesions have been described in the literature ${ }^{(5)}$. These lesions present numerous challenges in their diagnosis and management and hence are relevant to the practice of most otolaryngologists. We present our experience with three pterygopalatine fossa lesions and our review of the relevant literature.

\section{Methodology}

We reviewed three case studies of patients with pseudotumours of the pterygopalatine fossa presenting to the Department of Otolaryngology - Head \& Neck Surgery, Freeman Hospital, Newcastle upon Tyne, UK. For literature review, a Medline search was performed using keywords: pseudotumour, skull base, head and neck. The results were filtered for human studies and English language literature. Ninety-eight studies were identified. Abstracts were searched manually for appropriate studies for this review. 

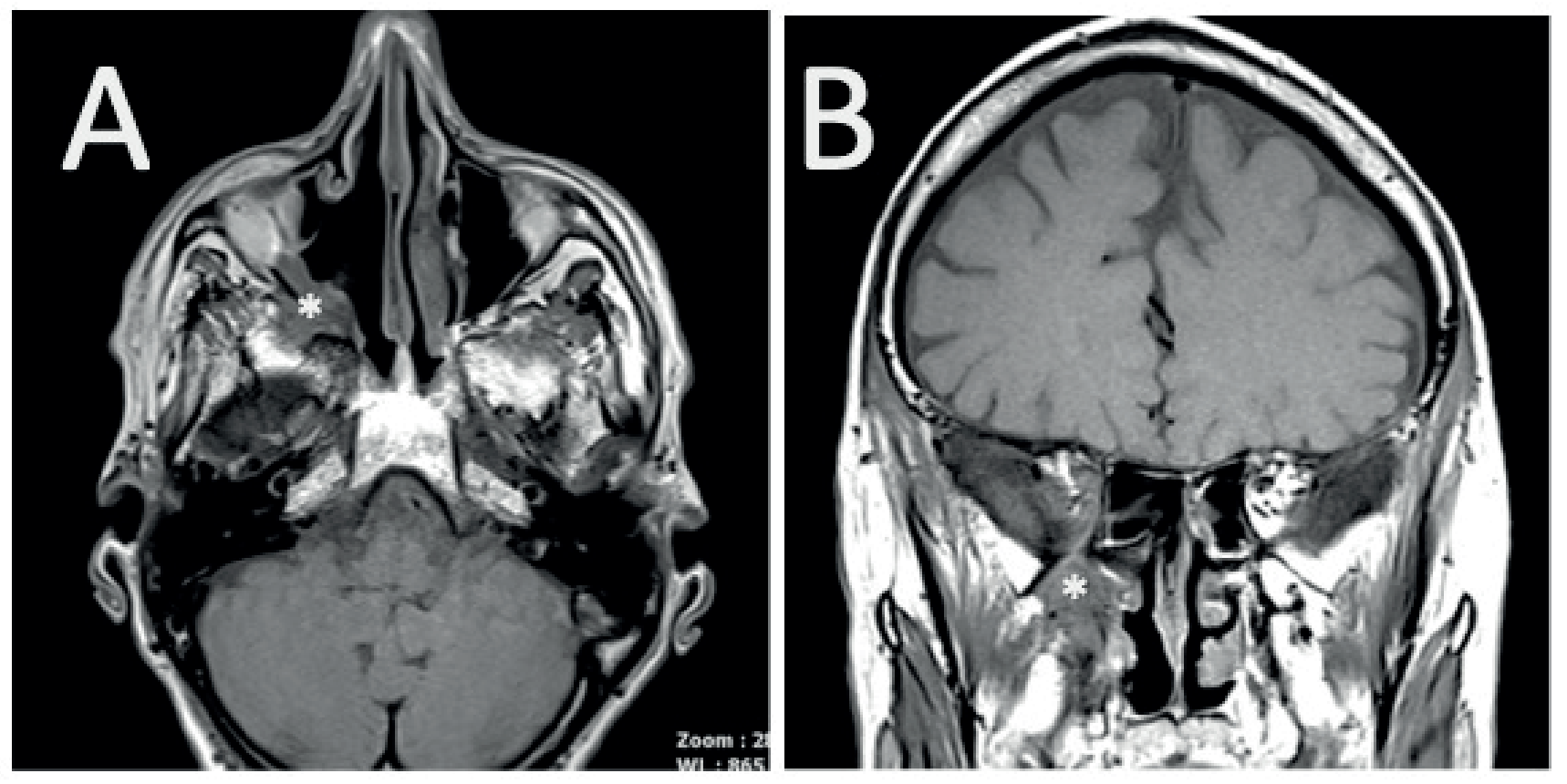

Figure 1. A.T1 W axial MRI showing hypointense lesion $\left(^{*}\right)$ in the right pterygopalatine fossa involving the right orbital apex. B. T1 W coronal MRI showing a hypointense lesion suggesting a combination of fibrosis and attenuated cellularity. These lesions may show marked Gadolinium enhancement.

\section{Case 1}

A 59-year-old male initially presented with flashes of light and reduced vision in the right eye to a local ophthalmology department. The symptoms improved spontaneously and the examination including intra-ocular pressure, fundoscopy and field test were unremarkable.

Two years later he again presented as an emergency to the ophthalmology and neurosurgical teams with sudden visual disturbance in the right eye, drooping upper eyelid and right retro-orbital pain radiating to the right maxillary region. He had previous medical history of ischaemic heart disease and had undergone coronary artery bypass graft. On examination he had partial right ptosis, restricted adduction, elevation and depression on the right consistent with partial 3rd and 6th nerve palsies with pupillary sparing. All routine blood tests were normal. CT scan of the brain and orbit revealed a mass in the right pterygopalatine fossa. MRI of the paranasal sinuses showed extension of the disease process into posterior orbit (Figure 1). The patient was referred to the ENT department where fullness at the posterior end of middle turbinate was noted. A transnasal approach was used for biopsy and debulking. The procedure was uneventful and the biopsies showed fibrous tissue containing patchy chronic inflammation. Mixture of small lymphocytes, plasma cells and histiocytes were seen. The fibrous tissue was paucicellular and densely hyalinised. Immunohistochemistry (CD79a, CD3, IgG and lgG4) showed few inflammatory cells. There was no evidence to suggest malignancy or lgG4 related disease process. There was further deterioration of visual acuity and retro-orbital pain and the examination revealed pale right optic disc and impaired colour vision to Ishihara chart testing. The patient was diagnosed with pseudotumour of the right pterygopalatine fossa with right orbital apex syndrome. Prednisolone was commenced at $60 \mathrm{mg}$ daily for two weeks. Pain improved initially but the vision deteriorated further with profound colour vision loss in the right eye. The steroids were reduced to $10 \mathrm{mg}$ daily with tapering dose of $2 \mathrm{mg}$ daily over 6 weeks. At his last appointment 6 months after his initial presentation there was no further deterioration of his vision and was pain free. Further care is on-going.

\section{Case 2}

A 37-year-old male initially presented with left facial pain with occasional blurring of vision and diplopia. On examination his visual acuity was $6 / 5$ on the right and $6 / 6$ on the left. He had slight restriction of left eye movements and mild ptosis. The rest of examination including intra-ocular pressure, fundoscopy, Goldman visual field test and colour vision were normal. His routine blood tests including inflammatory markers were normal. CT scan revealed a left pterygopalatine fossa lesion (Figure 2). A Caldwell Luc approach was used to biopsy the lesion. The biopsy showed features consistent with an inflammatory sclerosing process. After a debulking procedure there was significant improvement in his symptoms and ptosis. 5 months later he again developed pain around his left eye but ocular examination was 

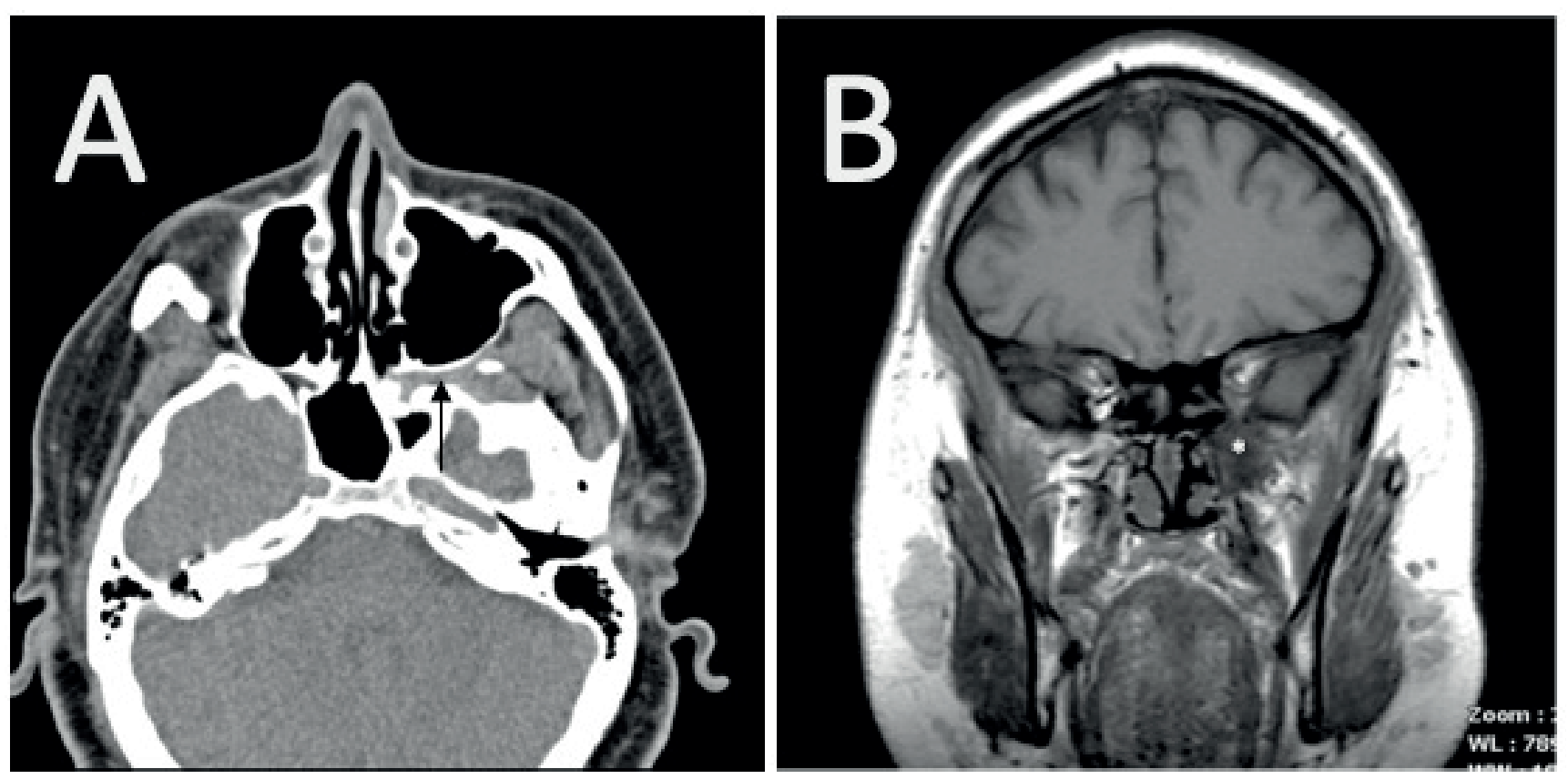

Figure 2. A. Axial CT scan showing homogeneous lesion in the left pterygopalatine fossa (black arrow). B. T1 W coronal MRI confirming hypointense lesion (Asterix) in left pterygopalatine fossa extending into left orbit. Hypointensity on T1 and T2 images helps differentiate IP from skull base neoplasms which are characteristically iso or hyperintense.

normal. Two months later the pain got worse and started to radiate to the maxillary area. His visual acuity had reduced to $6 / 12$ in his left eye. He developed slight restriction of his extra-ocular movements and there was mild ptosis. Optic nerve function was intact. The patient declined the offer of systemic steroids. 3 months later there was worsening of his headache and facial pain. His visual acuity had deteriorated to 8/18-1 on the left side. He also had developed central scotoma with enlarged blind spot and relative afferent pupillary defect. The colour vision had also reduced. An MRI scan was repeated which showed progression of disease. Intravenous steroids were commenced followed by a course of oral Prednisolone. There was no improvement with systemic steroids after a week and he was commenced on Azathioprine 50mg twice daily and Prednisolone was continued with $40 \mathrm{mg}$ daily. The visual acuity was reduced to finger counting at 1 meter only. The painful ophthalmoplegia got significantly worse and left medial wall orbital decompression was attempted for symptom relief. The ptosis improved but the pain persisted. His visual acuity was measured at $6 / 60$ in the left eye. The patient had no evidence of residual disease or progression on his repeat MRI scan 1 year after his decompression procedure.

\section{Case 3}

A 50 years old male presented with 8 weeks history of left sided eye pain radiating to face, crackling sound in left ear and intermittent dizziness. On examination he had a pupillary sparing left 3rd nerve palsy and proptosis. The rest of the ear, nose and throat examination including endoscopy was normal. CT and MRI revealed a mass in the left pterygopalatine fossa extending into infratemporal fossa, left cavernous sinus and abutting the left optic nerve. There was no bone involvement, MRI short tau inversion recovery (STIR) sequence showed no enhancement. Radiological findings were consistent with fibrosis.

Autoantibodies screen, Immunoglobulins, plasma electrophoresis, acetylcholine esterase were all normal. Chest X-ray was also unremarkable.

A Caldwell Luc approach was used to biopsy the lesion. It was noted that fat in the pterygopalatine fossa was replaced by a solid mass of tissue with areas of hard candlewax consistency. Histology confirmed idiopathic fibrotic changes only. There were no adverse features. The biopsy was repeated but again showed similar changes.

The patient was started on high dose corticosteroids for 4 weeks, which brought significant symptom control. The visual impairment improved as well and the corticosteroids were tapered over the next three weeks and then continued at 15 milligrams once daily. The total course was for five months. An MRI three months later showed improvement but no resolution. A third follow up scan was arranged 6 months later, which remained the same. At his last follow up 12 years later the patient remained asymptomatic and there was no further progression of the disease. 


\section{Discussion}

Idiopathic pseudotumour or inflammatory pseudotumours involving the skull base are rare. However, due to their anatomic location and considerable morbidity they are extremely important. In contrast to the orbital variant, these are clinically more aggressive and present numerous challenges in their diagnosis and treatment ${ }^{(6)}$.

\section{Aetiology}

The exact cause is still unknown. Multiple theories about the pathogenesis have been postulated.

\section{a. Autoimmune reaction}

Some authors believe IP are caused by an autoimmune reaction mediated by both $B$ and T lymphocytes ${ }^{(1)}$. A subgroup of these patients has a type of lgG4 related sclerosing disease that can also present with autoimmune pancreatitis and sclerosing sialadenitis as well as IP(4).

\section{b. Infection}

An infectious process such as sinusitis or syphilis could trigger IP. This is true of some patients presenting with acute history of lesion developing with associated upper respiratory tract infections or other viral illnesses. However, no definitive organism has been reliably implicated in the disease process. Others believe that organisms like Epstein-Barr Virus (EBV), Human Herpes virus 8, Human immune deficiency virus (HIV) could be the trigger ${ }^{(1)}$.

\section{c. Fibroproliferative disorders}

The presence of clonal cytogenic abnormalities supports the theory of fibroproliferative neoplastic origin of these lesions ${ }^{(7)}$.

\section{d. Trauma}

The development of IP after instrumental manipulation has led some authors to believe that a dramatic tissue response in some patients lead to the formation of an exuberant inflammatory reaction with secondary tissue proliferation ${ }^{(8)}$.

\section{Presentation}

Presentation depends on the site of the lesion. Orbital pseudotumours usually present with painful ophthalmoplegia while extra-orbital lesions could present with cranial nerve palsies, facial pain, dysphagia, hearing loss, otalgia or nasal obstruction. Constitutional symptoms such as fever, anorexia, weight loss, malaise and somnolence have been reported in 15 to $30 \%$ of patients with $\mathrm{IP}^{(9)}$.

Cranial nerve palsies result from direct nerve compression or extensive primary neural involvement, which may be acute or sub-acute ${ }^{(4,9,10)}$.

\section{Associations}

The relationship between IP and diabetes has not been reported consistently. The increased susceptibility to infection with an alteration of immunity in diabetic patients might be one possible explanation ${ }^{(6)}$.

Laurenzo and Graham noted that $23 \%$ of patients develop fibrosclerosing lesions at other sites including retroperitoneal fibrosis, orbital pseudotumour, sclerosing cholangitis and mediastinal fibrosis ${ }^{(11)}$. They recommended routine screening for these lesions with liver function tests and CT chest and abdomen.

\section{Diagnostic evaluation}

For any head and neck IP a thorough ear, nose and throat examination should include the neck, cranial nerves and ophthalmological assessment. Diagnosis is based on the histological features in light of clinical, laboratory and radiological findings.

As part of routine workup, the following investigations should be considered:

\section{a. Radiology}

The radiological features of IP are nonspecific and not characteristic $^{(12)}$. MRI is the modality of choice. In most, lesions will be hypointense on $\mathrm{T} 1$ and $\mathrm{T} 2$ weighted images and show marked Gadolinium enhancement. The hypointensity seen on $\mathrm{T} 2$ images likely reflects a combination of fibrosis and attenuated cellularity ${ }^{(10)}$. Enhanced CT may show homo- or heterogeneity and hypo, iso- or hyperdensity. Ultrasound also shows variable pattern of echogenicity with ill-defined margins in aggressive lesions. Doppler ultrasound may show prominent vascularity ${ }^{(9)}$. Sinonasal IP have more aggressive appearance with bony changes including erosion, remodelling and sclerosis ${ }^{(12)}$. IP lesions of the skull base show marked T2 hypointensity which helps differentiate them from skull base neoplasms that typically show iso to hyperintensity on $\mathrm{T} 2$ weighted images. However, these features cannot distinguish IP from skull base osteomyelitis, paragangliomas, neuromas and other granulomatous disorders e.g. granulomatosis with polyangiitis and Langerhans cell histiocytosis ${ }^{(13)}$.

PET-CT is sometimes considered, which shows a hypermetabolic lesion ${ }^{(14)}$. Chest X-ray might be useful to rule out synchronous lung lesion and sarcoidosis ${ }^{(5)}$.

\section{b. Open biopsy}

Depending on the site of lesion, open biopsies may be challenging. Often multiple biopsies are necessary to rule out other malignant neoplasms and inflammatory conditions ${ }^{(15)}$. The biopsy may be attempted using transoral, transcervical or an endoscopic approach. Endoscopic approach should be considered first because of low morbidity. In our patients, we used combined endoscopic transnasal-transmaxillary and Caldwell 
Luc approach to the pterygopalatine fossa.

\section{c. Histology}

The characteristic histopathological features include mixed inflammatory infiltrate with polyclonal lymphocytes, macrophages, polymorphonuclear cells, plasma cells, eosinophils and histiocytes with variable fibrosis. Immunohistochemical stains are used to identify cell lines and help in the grading process. Histopathological grading is based on the variability of cellular infiltrate, stromal components, vascular changes and tissues involved. The three main categories include the lymphoid, granulomatous and sclerosing variety ${ }^{(1)}$. Others divide these lesions into two main types:

a) Sclerosing type

b) Non-sclerosing type

i. Lymphoid

ii. Fibrotic

iii. Mixed

The sclerotic lesions are locally more aggressive and respond poorly to therapy. They are often associated with multifocal sclerotic disease processes. Some evidence suggests that the sclerosing variant respond to steroids if the disease duration has been brief ${ }^{(16)}$. Others believe that there is apparent relationship between the age of the lesion and degree of cellularity or fibrosis ${ }^{(17,18)}$.

There is a wide variation in the histological patterns and hence various synonyms exist to describe these lesions including plasma cell granuloma, mast cell granuloma, xanthogranuloma, histiocytoma, inflammatory myofibroblastic / myofibrohistiocytic proliferation.

\section{d. Blood tests}

Once biopsied, the suspicion of an inflammatory process would suggest further tests including erythrocyte sedimentation rate (ESR), anti-Nuclear Antibody, anti-double stranded DNA, anti-Neutrophil Cytoplasmic Antibody (cANCA and pANCA), Respiratory Protein-R and serum protein electrophoresis. Syphilis serology and testing for HIV could be helpful in certain cases.

\section{Treatment}

\section{Corticosteroids}

High dose corticosteroid is the treatment of choice for orbital IP. The dramatic response seen in orbital lesions within 24 to 72 hours is often considered a diagnostic test for orbital IP $P^{(1)}$. Maombaerts et al. found that $78 \%$ of patients treated with high dose corticosteroids had an initial response but achieved cure in $37 \%$ only. $52 \%$ had disease recurrence ${ }^{(20)}$. Other studies have noted a response rate of approximately $80 \%$ achieving remission in $40-50 \%$ of patients. Recurrence after cessation of treatment occurs in approximately $20 \%$ of cases $^{(4)}$. Extra orbital IP have a variable response to high dose corticosteroids as demonstrated by our patients. Poor response is seen in lesions with high degree of fibrosis or sclerosis. Lesions with abundant lymphocytes and plasma cells are considered more sensitive. This feature has been observed in both orbital and extra orbital lesions ${ }^{(1,4,7,16,19)}$. Weisman et al. ${ }^{(19)}$ and Osguthorpe et al. ${ }^{(21)}$ observed that the IP appear less likely to respond to steroids if the lesion has a more chronic course.

There is no standard dose or duration. Some authors recommend using low dose steroids for 6 months to prevent recurrence or relapse ${ }^{(4,6)}$.

Mangiardi et al. treated all their six patients with skull base IP with Prednisolone $1 \mathrm{mg} / \mathrm{kg} /$ day maximum $60 \mathrm{mg}$ until symptom resolution and normalization of imaging studies ${ }^{(1)}$. The dose was then tapered over 2 weeks. The duration of treatment ranged from 10 to 60 days prior to tapering. Three patients showed complete response while one had partial response but the disease was stable. One patient needed adjuvant chemotherapy with cyclophosphamide to stabilise the disease. One patient had a recurrence, which was treated again with corticosteroids.

\section{Surgery}

Surgical resection can be considered as an alternative treatment option ${ }^{(6)}$. Ruaux and Williamson recommend surgery as first line treatment for lesions involving paranasal sinuses and the temporal bone $\mathrm{e}^{(8,22,23)}$.

If a lesion is easily accessible and can be excised completely with low risk of complications, most surgeons would consider surgery. However, in lesions of the skull base with cranial nerve palsies complete resection is difficult. More aggressive surgery carries high risk of functional and cosmetic defects. Therefore, the risk of surgery should be weighed up against the risk of complications and discussed with the patient.

\section{Radiation}

Radiation treatment is considered for patients resistant to corticosteroid therapy or those who are unable to tolerate corticosteroids. However, some authors question the role of radiotherapy in steroid resistant cases ${ }^{(6)}$. The response to radiation therapy is variable with sclerosing and granulomatous varieties responding poorly ${ }^{(1)}$. There is no clear consensus on the dose of radiation therapy. However most recommend using low dose radiation of less than 10 Gy for orbital IP.

Lee et al. assessed the response of skull base IP to steroids and radiation therapy. Their study showed poor response to both high dose corticosteroids and low-dose radiation therapy ${ }^{(6)}$. They suggested that more aggressive approach should be used in the treatment of aggressive skull base IP with high dose radiation with or without concurrent corticosteroids. 


\section{Chemotherapy}

Chemotherapeutic agents have shown modest success in steroid responsive patients. Agents used include azathioprine, cyclophosphamide and cyclosporine. Our patient who did not show any response to steroids initially, eventually responded well with an azathioprine and corticosteroid combination.

\section{Multi-modality}

Most extra orbital IP require multi-modality treatment. Patients who initially are steroid resistant can have significant clinical and radiological response to steroids after surgical decompression. ${ }^{(10,16)}$. A combination of cyberknife radiosurgery and rituximab, an anti-CD20 chimeric murine/human monoclonal antibody was used in a steroid resistant patient with good effect ${ }^{(4,16)}$. Brannan advocated using $2 \mathrm{mg} / \mathrm{kg}$ oral prednisolone or intravenous methylprednisolone as initial treatment followed by radiotherapy and / or surgical debulking ${ }^{(24)}$. Some have noted that incomplete surgical excision may result in worsening of symptoms caused by reactivation of the immune response. However, others argue that partial excision may provide significant symptomatic relief secondary to an inflammatory 'burnout' phenomenon ${ }^{(16)}$.

Cross et al. described a case of 53-year-old female with IP of the left submandibular gland, which was initially treated with surgery ${ }^{(17)}$. Due to severe fibrosis and extension into skull base, carotid sheath and inferior border of the mandible, limited excision was performed and the patient was treated with high dose steroid post operatively. The patient had a good response but the disease recurred after cessation of treatment. She was treated again with low dose steroids and showed complete response.

\section{Prognosis}

Due to the relative rarity of significant case series or studies it is difficult to generalise about prognosis. However, it does appear to be linked to its histopathological and cellular classification as well as clinical presentation. IP may show a tendency toward spontaneous regression ${ }^{(9)}$.

In some cases, the disease process progresses to and can lead to general deterioration of health leading to death. However, so far, IP has not been reported as direct cause of death ${ }^{(6)}$.

Several cases in the literature found lymphoma in cases of IP after a chronic course $\mathrm{e}^{(9)}$. It is unclear whether these were missed diagnosed lesions or a genuine transformation within IP. In theory there is a risk of malignant transformation in lesions that show ganglion-like cells, p53 expression and aneuploidy but in the head and neck region there have been no reported cases of malignant change $\mathrm{e}^{(7,25)}$.

\section{Conclusion}

Idiopathic pseudotumours of the anterior skull base, although rare, present significant issues in their management. It can both clinically and radiologically mimic a neoplasm. Most of the information is based on the orbital pseudotumours but the extra-orbital and especially skull base IP is more aggressive and respond differently to conventional therapies. The literature is sparse on skull base IP but through case reports and series like ours we can improve our understanding of their behaviour and learn how to manage them effectively.

\section{Acknowledgement}

Not applicable

\section{Authorship contribution}

SC proposed the concept and structure of the manuscript. MKK conducted case reviews, literature review and completed the initial draft of the manuscript. All authors read and approved the final manuscript.

\section{Conflict of interest}

None to declare.

\section{Ethics approval and consent to participate} Not applicable.

\section{Consent for publication}

Written informed consent for publication of their clinical details and clinical images was obtained from the patients. A copy of the consent form is available for review by the Editor of this journal.

\section{Availability of data and materials}

Not applicable.

\section{Funding}

Not applicable.
1. Mangiardi JR, Har-El G. Extraorbital skull base idiopathic pseudotumor. Laryngoscope. 2007;117(4):589-94.

2. Lee RG, Weber DE, Ness AB, Wasman JK, Megerian CA. Inflammatory pseudotumor of the middle ear masquerading as Bell's palsy. Am J Otolaryngol. 2007;28(6):423-6.

3. Coffin CM, Watterson J, Priest JR, Dehner LP. Extrapulmonary inflammatory myofi- broblastic tumor (inflammatory pseudotumor). A clinicopathologic and immunohistochemical study of 84 cases. Am J Surg Pathol. 1995:19(8):859-72.

4. Maruya S, Miura K, Tada Y, Masubuchi T, Nakamura N, Fushimi C, et al. Inflammatory pseudotumor of the parapharyngeal space: a case report. Auris, nasus, larynx. 2010;37(3):397-400
5. Lee JH, Jung MK, Song CE, Yeo SW, Lee HK Yang PS, et al. Concomitant inflammatory pseudotumor of the temporal bone and lung: a case report. ENT J. 2007:86(10):614-6.

6. Lee DK, Cho YS, Hong SH, Chung WH, Ahn YC. Inflammatory pseudotumor involving the skull base: response to steroid and radiation therapy. Otolaryngol Head Neck Surg. 2006 Jul;135(1):144-87. 
7. Van Weert S, Manni JJ, Driessen A Inflammatory myofibroblastic tumor of the parotid gland: case report and review of the literature. Acta oto-laryngologica. 2005;125(4):433-7.

8. Hadi U, el-Bitar M, Zaatari G. Postadenoidectomy inflammatory pseudotumor. Rhinology. 2001;39(3):176-9.

9. De Vuysere S, Hermans R, Sciot R, Crevits I Marchal G. Extraorbital inflammatory pseudotumor of the head and neck: CT and MR findings in three patients.AJNR Am J Neuroradiol. 1999;20(6):1133-9.

10. Seol JG, Loevner LA, O'Malley BW, Jr., Grady MS. Inflammatory pseudotumor of the trigeminal nerve: a neoplastic mimic you do not want to miss. AJNR Am J Neuroradiol. 2009;30(10):1941-3.

11. Laurenzo JF, Graham SM. Tumefactive fibroinflammatory lesion of the head and neck: a management strategy. ENT J. 1995;74(2):8791.

12. Park SB, Lee $J H$, Weon YC. Imaging findings of head and neck inflammatory pseudotumor. AJR Am J Roentgenol. 2009;193(4):1180-6.

13. Curry JM, King N, O'Reilly RC, Corao D Inflammatory pseudotumor of the inner ear: are computed tomography changes pathognomonic? Laryngoscope. 2010;120(6):1252-5
14. Zitsch RP, 3rd, Pollak N, Loy TS. Management of inflammatory pseudotumor of the larynx. Otolaryngol Head Neck Surg. 2007;136(1):139-41.

15. Lee HM, Choi G, Choi CS, Kim CH, Lee SH. Inflammatory pseudotumor of the maxillary sinus. Otolaryngol Head Neck Surg. 2001;125(5):565-6.

16. Vivero RJ, Doshi PH, Eloy JA, GomezFernandez C, Casiano RR. Primary sclerosing fibroinflammatory pseudotumor of the maxillary sinus. ENT J 2011;90(12):578-90.

17. Cross DL, Switter DJ. Fibroinflammatory pseudotumor of the submandibular gland. Head Neck. 1996;18(5):465-8.

18. Williams SB, Foss RD, Ellis GL. Inflammatory pseudotumors of the major salivary glands. Clinicopathologic and immunohistochemical analysis of six cases. Am J Surg Path. 1992;16(9):896-902.

19. Weisman RA, Osguthorpe JD. Pseudotumor of the head and neck masquerading as neoplasia. Laryngoscope. 1988;98:610-4.

20. Mombaerts I, Schlingemann RO, Goldschmeding R, Koornneef L. Are Systemic Corticosteroids Useful in the Management of Orbital Pseudotumors? Ophthalmology. 1996;103(3):521-8.

21. Osguthorpe JD, Saunders RA, Adkins WY Evaluation of and access to posterior orbital tumors. Laryngoscope. 1983;93(6):766-71.
22. Williamson RA, Paueksakon P, Coker NJ. Inflammatory pseudotumor of the temporal bone. Otol Neurotol. . 2003;24(5):818-22.

23. Ruaux C, Noret P, Godey B. Inflammatory pseudotumour of the nasal cavity and sinuses. J Laryngol Otol. 2001;115(07):563-6.

24. Brannan PA. A review of sclerosing idiopathic orbital inflammation. Curr Opin Ophthalmol. 2007;18(5):402-4.

25. Amir R, Danahey D, Ferrer K, Maffee $M$ Inflammatory myofibroblastic tumor presenting with tracheal obstruction in a pregnant woman. Am J Otolaryngol. 2002;23(6):362-7.

Muhammad Kamaal Khan

Department of ENT

Freeman Hospital

Newcastle upon Tyne, NE77DN

United Kingdom

Tel: +44-(0)191 2231413

Fax: +44-(0)191 2231246

E-mail: kamaalkhan@hotmail.com

ISSN: 2589-5613 / ○2019 The Author(s). This work is licensed under a Creative Commons Attribution 4.0 International License. The images or other third party material in this article are included in the article's Creative Commons license, unless indicated otherwise in the credit line; if the material is not included under the Creative Commons license, users will need to obtain permission from the license holder to reproduce the material. To view a copy of this license, visit http://creativecommons.org/licenses/by/4.0/ 